\title{
Philosophiques
}

\section{Analyse critique de quelques modèles sémiotiques de l’idéologie (première partie)}

\section{Robert Tremblay}

Volume 17, numéro 1, printemps 1990

URI : https://id.erudit.org/iderudit/027105ar

DOI : https://doi.org/10.7202/027105ar

Aller au sommaire du numéro

Éditeur(s)

Société de philosophie du Québec

ISSN

0316-2923 (imprimé)

1492-1391 (numérique)

Découvrir la revue

Citer cet article

Tremblay, R. (1990). Analyse critique de quelques modèles sémiotiques de l'idéologie (première partie). Philosophiques, 17(1), 71-112.

https://doi.org/10.7202/027105ar
Résumé de l'article

Ce texte s'adresse au problème de l'analyse sémiotique de la question de l'idéologie. Il montre la faiblesse des principaux dévelop- pements traditionnels sur ce point. Dans cette première partie, nous rejetons les théories des sémioticiens saussuriens (Hjelmslev, Barthes et Greimas), en raison de leur conception du langage et de la connotation. Dans la seconde partie, nous analyserons les théories sociosémiotiques de Kristeva, Morris et Eco. En conclusion, nous montrerons la pertinence d'une approche peircienne de cette problématique. 


\title{
ANALYSE CRITIQUE DE QUELQUES MODĖLES SÉMIOTIQUES DE L'IDÉOLOGIE (première partie)
}

\author{
par Robert Tremblay
}

\begin{abstract}
RÉSUMÉ. Ce texte s'adresse au problème de l'analyse sémiotique de la question de l'idéologie. Il montre la faiblesse des principaux développements traditionnels sur ce point. Dans cette première partie, nous rejetons les théories des sémioticiens saussuriens (Hjelmslev, Barthes et Greimas), en raison de leur conception du langage et de la connotation. Dans la seconde partie, nous analyserons les théories sociosémiotiques de Kristeva, Morris et Eco. En conclusion, nous montrerons la perrinence d'une approche peircienne de cette problématique.
\end{abstract}

ABSTRACT. This paper deals with the problem of analysing the question of ideology as a semiotic one. It shows the weakness of the main traditional developments on this issue. In this first part, the theories of saussurian semioticians (Hjelmslev, Barthes et Greimas) are rejected, because of their conception of language and connotation. In the second part, we will analyse the sociosemiotic theories of Kristeva, Morris and Eco. In conclusion, we will show the relevance of a peircean approach of this problematic.

Les problèmes fondamentaux rencontrés par les théories de l'idéologie ne peuvent être adéquatement posés, et par là même résolus, en dehors d'une perspective sémiotique. Cette affirmation pour le moins tranchée n'aura guère l'heur de plaire à ceux qui se consacrent au développement d'une théorie essentiellement sociopolitique de l'idéologie. C'est l'une des raisons pour lesquelles il faudrait la fonder bien mieux que nous ne serons en mesure de le faire ici. Cela ne plaît guère non plus aux sémioticiens, du moins à certains d'entre eux, pour qui un objet aussi "évanescent» que l'idéologie ne saurait satisfaire aux conditions définitoires d'une 
articulation sémiologique rigoureuse. C'est dire les difficultés immédiatement prévisibles que toute sociosémiotique doit s'attendre à devoir surmonter pour reconstruire au sein de sa problématique un objet tel que l'idéologie.

\section{Le champ sémiotique}

Une première définition approximative présenterait la sémiotique ${ }^{1}$ comme la discipline concernée par les systèmes symboliques ; ou encore comme la théorie générale des systèmes de signes. On distingue quelquefois la sémiologie de la communication et de la signification, mais cette distinction ne peut avoir de pertinence que du point de vue de l'école dite de « sémiologie de la communication " ${ }^{2}$, puisque cette dernière veut exclure du champ sémiotique tout processus de production de signes qui ne soit engager dans l'intention explicite et communément reconnue de communiquer. Cette exclusion est inacceptable parce qu'elle mène au rejet de tous les phénomènes de signification non transparente où les intentions du destinateur sont obscures ou contradictoires ${ }^{3}$. On pourrait définir plus précisément la sémiotique comme la science des processus de signification et de communication ou science des sémiosis.

1. "Sémiotique " ou « sémiologien ? : sauf exception explicite, nous utiliserons les deux termes comme des équivalents, bien que nous préférions le terme « sémiorique \%, en raison de notre adhésion à la tradition peircienne. "Sémiologie" ayant éré proposée par Saussure, nous l'emploierons plus volontiers pour désigner les travaux de cette tradition, en autant que les auteurs eux-mêmes se qualifient de sémiologues. Aucune distinction théorique entre les deux concepts ne nous semble s'imposer ou encore rallier les chercheurs, comme celle par exemple qui propose de réserver « sémiologie * pour désigner la discipline générale, et sémiotiques pour les secteurs particuliers (ou vice-versa) ! Par contre, " sémiotique semble devoir s'imposer sur le long terme; il est déjà d'usage chez les chercheurs anglo-saxons, italiens, allemands, soviétiques et plusieurs français. C'est l'appellarion reconnue par l'Association internationale d'études sémiotiques. Il existe aussi un argument étymologique en faveur de notre choix: sémiotikê est déjà employé chez les anciens grecs pour désigner la sympromatologie médicale, et semiotik est repris par John Locke pour désigner le caractère symbolique de la connaissance (au livre IV, XXI, de : An Es say Concerning Humen Understanding, 1690) : par contre * sémiologie» est un néologisme introduit par Saussure dans son enseignement en 1908 ; il nous semble préférable de rerenir le terme qui a les racines étymologiques les plus profondes.

2. C'est, par exemple, la position de Georges Mounin, qui définit la sémiologie comme "la science générale de tous les systèmes de communication par signaux, signes ou symboles ». Introduction à la sémiologie, Paris, Minuit, 1970,248 p.; p. 7

3. Comme c'est très souvent le cas tel que la théorie de la communication paradoxale l'a montré. 
Nous utilisons pour qualifier la discipline sémiotique le qualificatif de "science»; c'est en l'occurrence un mot bien pompeux. Disons-le sans ambage, la sémiotique (ou, dans la tradition française, la sémiologie), dans son état actuel, et malgré son extrême fécondité, ne peut prétendre au qualificatif de science qu'à considérer la scientificité en un sens très large et peu rigoureux. ${ }^{4}$ On peut dénombrer plusieurs courants de pensée qui ont chacun leur vocabulaire, leur méthodologie, leur cadre explicatif ; dans la mesure où il n'existe de consensus ni sur son objet, ni sur ses méthodes, ni sur le statut de ses investigations, la recherche sémiotique relève encore de la pré-science et reste largement influencée par la philosophie. Cette situation d'ensemble de la discipline se complique encore lorsque la sémiotique se penche sur un objet aussi controversé que l'idéologie.

Nous examinons ici sommairement, et seulement dans la perspective de leur contribution à une théorie de l'idéologie, cinq approches du sémiotique qui, comme nous allons tenter de la montrer, ne constituent pas des réponses suffisantes aux questions impliquées dans la constitution de l'idéologie comme objet sémiotique mais contribuent, à un titre ou à un autre, à la problématique générale de la production sociale du sens. Nous chercherons dans les pages qui suivent à analyser et critiquer les principales théories de la sémiologie traditionnelle eu égard à la compréhension de l'idéologie comme artefact symbolique. Nous étudierons les courants suivants.

Dans la première partie de notre article:

- la sémiologie saussurienne qui passe par Hjelmslev, Barthes et Greimas ;

4. Sur ce point, nous nous rallions au critère socio-épistémologique proposé par Thomas Kuhn : l'existence d'un paradigme unificateur caractérise la science à sa maturité et la distingue de la pré-science. Il s'agit bien sûr là d'une condition nécessaire et non d'une condition suffisante. Les disciplines pré-scientifiques se distinguent par l'existence de plusieurs perspectives concurrentes et par une inter rogation constante sur les fondements, l'objet et les méthodes de la discipline. En ce sens, la sémiotique est bien dans un état pré-scientifique, bien qu'il y ait de nombreux prétendants au titre de paradigme: l'École de Paris, la sémiologie saussurienne, la sémiologie de la communication, la sémanalyse, la sémiotique peircienne, la sémiotique béhavioriste, etc. Nous proposons de qualifier ces prétendants, de pseudo-paradigmes. Il ne suffit pas de tenter une synthèse, ou un rapprochement pour établir un premier paradigme. (Référence : $L a$ structure des révolutions scientifiques, Paris, Flammarion, 1983, (1970), 284 p.) 
- la sémiologie de la communication qui est en fait une variante du saussurisme, elle commence avec Buyssens et se développe avec $L$. Prieto et $G$. Mounin.

Et dans la seconde partie :

- la sémanalyse qui est aussi une sémiologie hjelmslévienne mais très fortement influencée par la psychanalyse et le matérialisme historique; elle est l'œuvre personnelle de Julia Kristeva ;

- la sémiotique behavioriste et positiviste qui est associée au noms de Charles Morris;

- et enfin la sémiotique des codes du célèbre sémioticien italien Umberto Eco, laquelle est une tentative encyclopédiste de synthèse des différents courants en vue d'une unification disciplinaire.

Notre examen de toutes ces théories poursuit un but unique qu'il importe de préciser d'emblée : dégager l'apport spécifique de la sémiotique à l'étude des idéologies. Cela implique un double mouvement: il faut d'abord expliciter chaque théorie afin de dégager son intérêt pour une sociosémiotique de l'idéologie, puis il faut évaluer la contribution explicite de chaque théorie à la compréhension de notre objet. Enfin il faut voir, au cas à cas, s'il y a une possibilité de retenir certains apports de ces théories en vue de la constitution de la sociosémiotique comme discipline relativement autonome. Voilà ce à quoi nous voulons contribuer ici.

Avant d'aller plus loin, il est important d'expliquer les raisons de la distinction que nous suggérons de faire entre la sémiotique et la sociosémiorique. Nous postulons une différence de principe entre la sociosémiotique et les autres parties de la sémiotique générale, non pour dessiner une frontière infranchissable mais af in de bien délimiter l'objet propre de cette dernière branche disciplinaire de la sémiotique. En gardant en tête les limites de toute analogie, on peut se référer aux rapports entre linguistique et sociolinguistiques. Si la linguistique a pour objet la nature du

5. Sur la sociolinguistique, nous nous référons à l'excellent ouvrage de Jean-Baptiste MARCELLESI et Bernard GARDIN, Introduction à la sociolinguistique. La linguistique sociale, Paris, Larousse, 1974, $263 \mathrm{p}$. Sur la linguistique nous nous référons à Adrian AKMajIAN, Richard A. DEMERS, et Robert M. HARNISH, Linguistics. An Introduction to Language and Communication, Cambridge, M.I.T. Press, 1984, 547 P. 
langage et de la communication linguistique et aussi, il ne faut pas l'oublier, les diverses langues du monde, la sociolinguistique pour sa part étudie le caractère spécifiquement social du langage, les variations dialectales, l'évolution historique des langues et les codes sociosémantiques. Ainsi existe-il aussi une psycholinguistique, une phonologie, etc. Les rapports sont similaires entre la sémiotique générale - théorie générale de la signification et de la communication par signes - et ses différentes branches: sémiotique visuelle, sémiotique musicale, psychosémiotique, sociosémiotique, etc. L'insistance sur le caractère social des systèmes symboliques n'entraîne pas une (con)fusion de perspectives; elle contribue plutôt à asseoir la validité de la démarche sociosémiotique. Aussi considérons-nous que la distinction entre sémiotique et sociosémiotique doit être maintenue, même si, comme nous le pensons, la sociosémiotique doit être une partie très importante de la sémiotique générale. Cependant l'analogie s'arrête ici. En effet, Labov affirme :

La plupart des composantes de la structure linguistique sont des règles tout à fait abstraites et tout à fait catégoriques - elles sont indépendantes des influences sociales, sans connotations subjectives, ne reflètent pas les stratifications ethniques, de classes ou de castes et ne relèvent pas de variations stylistiques. Elles sont absolument invariantes. (...) Mais... Je me représente volontiers la structure linguistique comme ouverte aux influences sociales sur un petit nombre de points spécifiques qui sont variables - certains de ces points sont utilisés par les linguistes en tant que variables sociales et peuvent pénétrer dans la structure sociale. ${ }^{6}$

Or le statut d'une véritable sociologie du langage étant rien moins que clairement établi ${ }^{7}$, nous sommes autorisés à comprendre la sociolinguistique actuelle essentiellement comme l'étude de variances socialement déterminées en rapport avec une norme linguistique que, par ailleurs, la grammaire générative et le structuralisme linguistique font profession d'analyser. La situation est cependant très différente avec la sémiotique, puisque nous n'avons pas d'assurance ferme quant à l'identification de normes sémiotiques de génération des séquences signifiantes. En outre, si de telles normes existent, comme on peut le penser, rien n'indique qu'elles

6. Dans J.A. Flshman, Advances in the Sociology of Language, tome 1, La Haye, Mouton, 1971, p. 496. Cité et traduit par Marcellesi, Gardin, op. cit., pp. 144-145.

7. Si Marcellesi et Gardin en tracent les contours, et mentionnent quelques études, l'essentiel des travaux sociolinguistiques qu' ils répertorient s'accorde avec l'affirmation de Labov. 
ont un caractère et une forme semblables aux normes linguistiques et qu'elles n'incluent pas d'emblée des variables sociologiques, psychologiques ou historiques. C'est en ce sens qu'il faut considérer sérieusement l'importance de la sociosémiotique - mais aussi de la psychosémiotique, de la sémiotique visuelle, etc. - dans le processus de constitution scientifique de la sémiotique générale elle-même.

Si la sémiotique ressemble tant à cette auberge espagnole que tous abhorrent (mais est-ce là une attitude bien raisonnable pour des sémioticiens?), on peut aisément douter de la pertinence d'un déplacement du coeur de la problématique de l'idéologie vers cette discipline ; d'autant que les récents travaux des sociologues, pensons à Pierre Ansart ou à Raymond Boudon par exemple, parviennent à des résultats intéressants sur une base beaucoup plus assurée (la sociologie). En fait une seule conviction peut justifier une opération aussi problématique : la croyance en la nécessité théorétique d'opérer ce déplacement. Nous contribuerons en conclusion à l'affermissement de cette croyance.

\section{La «filière » saussurienne}

Notre premier cas d'espèce est assurément le mieux connu, entre autres raisons, à cause de l'importance des travaux de Roland Barthes. De la même façon que, dans une certaine tradition marxiste, on subordonnait l'instance idéologique à cette autre instance considérée comme plus fondamentale, l'économique, dans la filière saussurienne le social n'apparaît jamais que dans une position de secondarité, mais cette fois-ci par rapport à la langue conçue comme un pur système de dénotation. Un exemple parmi d'autres, Barthes écrit à propos du discours mythique ${ }^{8}$ :

On le voit, il y a dans le mythe deux systèmes sémiologiques, dont l'un est déboîté par rapport à l'autre : un système linguistique, la langue (...) que j'appellerai langage-objet, parce qu'il est le langage dont le mythe se saisit pour construire son propre système ; et le mythe lui-même que j'appellerai méta-langage, parce qu'il est une seconde langue, dans laquelle on parle de la première. ${ }^{9}$

8. Est-il besoin de préciser que pour nous le mythe est une forme élémentaire d'idéologie?

9. Roland Barthes, Mythologies, Paris, Seuil, 1957, p. 200. 
Ici métalangage, ailleurs code ou connotation, le social ne pointe jamais à l'horizon du sémiologique autrement que sous les figures de la secondarité. Un peu comme si la communauté humaine n'était ni à l'origine, ni au principe de l'existence des systèmes de signes, mais n'en était que la causa adductiva. ${ }^{10}$

\section{FERDINAND DE SAUSSURE}

On doit pourtant reconnaître que Saussure considère que « le langage est un fait social ». ${ }^{11}$ Mais il considère également que la sémiologie doit être une branche de la psychologie:

Nous venons de voir que la langue est une institution sociale; mais elle se distingue par plusieurs traits des autres institutions politiques, juridiques, etc. Pour comprendre sa nature spéciale, il faut faire intervenir un nouvel ordre de faits.

La langue est un système de signes exprimant des idées, et par là, comparable à l'écriture, à l'alphabet des sourds-muets, aux rites symboliques, aux formes de politesse, aux signaux militaires, etc., etc. Elle est seulement le plus important de ces systèmes.

On peut donc concevoir une science qui étudie la vie des signes au sein de la vie sociale; elle formerait une partie de la psychologie sociale, et par conséquent de la psychologie générale; nous la nommerons sémiologie (du grec sémeîon, "signe»). Elle nous apprendrait en quoi consiste les signes, quelles lois les régissent. ${ }^{12}$

Remarquons premièrement que Saussure destine immédiatement cette nouvelle discipline à l'analyse du caractère social des systèmes de signes. Bien qu'il ait du social une conception présociologique ${ }^{13}$ et psychologiste, il est important de noter cette perspective sociosémiotique avant la lettre. Cependant les saussuriens font une distinction entre le langage et la langue. Si Saussure défend le concept général du caractère social de la langue, il ne l'en étudie pas moins d'abord comme un système : comme une structure abstraite de dénotation, exclusivement synchronique, et d'où la parole est exclue. Le système de la langue, c'est la langue arrachée

10. Chez Thomas d'Aquin, la causa adductiva se distingue de la causa principalis comme ce qui n'est que l'occasion contingente d'une autre cause essentielle. Ainsi Lalande le définit comme : «ce qui amène à l'acte la cause principale », p. 128.

11. Ferdinand de Saussure, Cours de linguistique générale, Paris, Payot, 1972, p. 21.

12. Ibid., p. 33 .

13. Au sens de Durkheim. 
du sol vivant de ses manifestations singulières et diachroniques. Car si la linguistique «a pour objet la langue, qui est sociale en son essence et indépendante de l'individu " ${ }^{14}$, il faut adopter une perspective essentiellement psychologique pour son appréhension. Cette caractérisation initiale ne saurait impliquer ce regard sur sa véritable nature sociale, par lequel la parole comme la langue apparaîtraient comme ordonnées par des déterminants proprement sociaux. Bref, Saussure a du social une conception telle que l'aveu de son caractère social n'entraîne qu'une conséquence limitée : une langue est un lexique sémantisé en vertu du principe de «l'arbitraire du signe ", combiné avec un ensemble de règles grammaticales communément partagées par une communauté humaine donnée. Saussure a une conception abstraite, "psychologique », du social. Ainsi il écrit : "Il n'y a donc rien de collectif dans la parole ; les manifestations en sont individuelles et momentanées. " ${ }^{15}$ On ne saurait imaginer, dans cette perspective, que la parole puisse dépendre d'autre chose que de la volonté individuelle. Ainsi, dès l'origine, on ne saurait concevoir que l'idéologie intervienne sur la langue autrement que de l'extérieur.

Une fois que l'on a expurgé la langue, désormais considérée comme un système totalement abstrait, de toute contamination sociale, et que l'on peut ainsi distinguer une linguistique interne (la linguistique en tant que telle), d'une linguistique externe, on peut affirmer tranquillement que «... la langue ne connaît que son ordre propre... ${ }^{16} \mathrm{Et}$ sous les espèces de la linguistique externe se trouvera alors réintroduit tout ce que l'on avait d'abord exclu: les rapports entre langue, race et civilisation, entre langue et histoire politique, entre langue et institutions et enfin entre langue et géographie (ou dialectologie). ${ }^{17}$ C'est de la même façon qu'au plan sémantique seront distinguées la dénotation, interne au système linguistique et première logiquement, de la connotation, externe et secondaire.

Le signe chez Saussure est une dyade : le signifiant est le signe perceptible, le signifié, son concept ${ }^{18}$. Par exemple le mot «arbre »

\footnotetext{
14. Ibid., p. 37.

15. lbid., p. 38 .

16. Ibid., p. 43.

17. Ibid., Pp. 40-41.

18. Op. cit., p. 99 et p. 144.
} 
est une certaine image acoustique: [arbr], c'est son signifiant; mais renvoie à un concept : ${ }^{19}$ c'est son signifié. La signification est la relation entre le signifiant et le signifié. Cette relation est à la fois immotivée et nécessaire une fois qu'elle est établie: c'est le principe de l'arbitraire du signe. La valeur du signe est constituée par cette relation en fonction du système auquel il appartient.

Pour l'école saussurienne, la dénotation du signe est sa fonction référentielle et sa représentation, l'image mentale que le signe suscite. ${ }^{20}$ Sa connotation est à la fois le sens secondaire qui s'attache à lui dans l'axe paradigmatique, et le «fourre-tout » des renvois non référentiels du signe. Dans son sens philosophique classique, la dénotation est la référence à un objet et la connotation, l'ensemble de ses attributs significatifs.

C'est sur cette double assise que s'assoie la conception sémiologique de subordination instancielle de l'idéologie : l'idéologie ne participerait ni du signe (signifiant et signifié), ni de la représentation, ni même de la dénotation. L'idéologie ne serait qu'une lointaine connotation, c'est-à-dire, pour parler philosophiquement, un quelconque attribut, d'ailleurs plus ou moins pervers, du signe.

Nous devons ici rompre le fil de l'exposé pour marquer notre profond désaccord avec cette conception. Non seulement la fonction référentielle n'est-elle jamais référentielle qu'à l'intérieur d'un inquestionné du renvoi à l'objet mais, de plus, le système auquel le signe appartient nécessairement est tout entier empreint d'une conception du monde particulière. Comme systèmes, tous les symbolismes sont connotés de manière immanente ; la dénotation de l'un n'étant jamais que la connotation de l'autre. C'est toujours par une certaine convention culturellement arbitraire que s'établit la signification signifiée et dénotée du signe ; ainsi le signe n'est-il assiégé par le connoté que sous le mode du retour du refoulé ou de la dérive du sens. Pour nous, l'idéologie ne peut être comprise dans un rapport de secondarisation, que ce soit par rapport à l'économique ou par rapport au linguistique : l'idéologie est bien dans le connoté, mais elle est aussi dans le dénoté, dans le référé, dans le représenté

19. Bien sûr, le petit dessin n'est pas le signifié « en personne » mais une autre représentation du signifié destinée à faire sentir le signifié sans recourir au langage verbal.

20. Oswald Duckot, Tzvetan ToDorov, Dictionnaire encyclopédique des sciences du langage, Paris, Seuil, 1972, p. 134. 
et dans le signifié; peut-être parce qu'elle est d'abord dans le signifiant. L'idéologie est toujours-déjà là parce que le pouvoir est lui-même toujours-déjà présent dans les signes. Certes il n'y est pas toujours manifeste, il faut que certaines conditions contextuelles soient remplies pour que la nature politique du signe se révèle : par exemple lorsqu'un performatif d'autorité est prononcé. Si le pouvoir est dans le signe, c'est que le signe est d'abord une action dans le monde qui exerce son propre pouvoir sur ce monde ${ }^{21}$.

\section{LOUIS HJELMSLEV}

Hjelmslev étaiera et radicalisera la position de Saussure. Or les travaux de Barthes doivent énormément, au plan théorique, à la pensée de Hjelmslev. Présentons à grands traits là où, dans sa théorie, les questions sociosémiotiques sont posées : c'est-à-dire essentiellement au niveau de la connotation. La dénotation d'un signe est un noyau stable de signification partagé par toute la communauté linguistique. La connotation, eile, échappe à la linguistique parce qu'elle n'est pas un fait de langue mais un fait de contexte, donc de parole et de détermination sociale du sens.

Pour Hjelmslev, les sémiotiques au sens propre sont composées de deux plans : un plan du contenu (le réseau des signifiés) et un plan de l'expression (le réseau des signifiants). Une sémiotique simple (ou système de signification) est une sémiotique dénotative. Il convient dans chaque cas de distinguer la forme (invariante) et la substance (variante) de chaque plan. L'analyse devra donc distinguer la forme de l'expression d'un signe (son invariant syntaxique) de sa substance (par exemple les sons ou les gestes par lesquels il est exprimé). De la même manière aurons-nous la forme du contenu, c'est-à-dire le rapport du signe à l'organisation formelle des signifiés en système et la substance du contenu qui désigne, selon Barthes, «les aspects émotifs, idéologiques, ou simplement notionnels du signifié, son sens positif $\gg{ }^{22}$ On voit donc que sur chacun des plans la forme (la structure d'organisation des formes) ne comporte qu'une dimension sociale extrêmement générale et abstraite, assimilable à un schème quasi-logique (d'ailleurs

21. Il est un effet interprétant, il est un sens.

22. "Éléments de sémiologie", in Le degré zéro de l'écriture, suivi de Éléments de sémiologie, Paris, Seuil, 1964, 181 p. ; p. 112. 
Hjelmslev le nomme justement "schéma linguistique» pour l'opposer à l'usage de la langue). Si la substance de l'expression renvoie à la phonétique, seule la substance du contenu évoque la signification (sociale) du signe. Ainsi la forme et la substance de l'expression et la forme du contenu sont-ils asociaux pour Hjelmslev. Seule la substance du contenu est susceptible de recevoir quelque détermination sociale, et encore pas dans un premier temps.

En effet, de prime abord une langue est une sémiotique dénotative, c'est-à-dire un système de mise en relation d'un système formel de signifiants avec un système de contenus dénotés dans une relation de signification. La signification émerge de cette mise en relation. On peut représenter ce rapport par le schéma suivant :

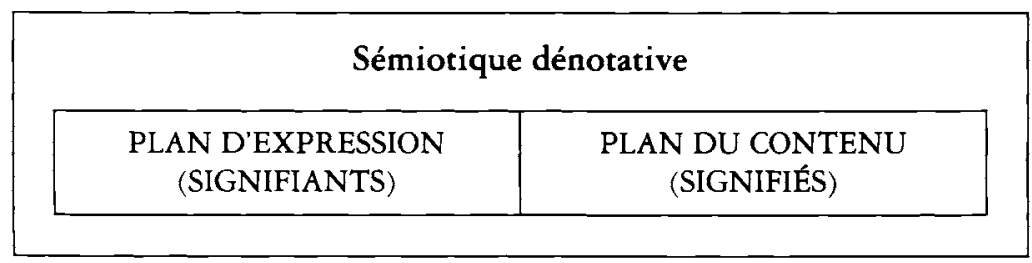

Mais le modèle se complexifie si l'on songe que chacun des deux plans peut être composé d'une sémiotique dénotative. Ainsi, les sémiotiques dont le plan du contenu est composé d'une sémiotique dénotative scientifique (une mise en relation scientifique d'un système formel avec un système de signifiés, son objet), c'està-dire obéissant à un critère d'empiricité, sont des métasémiotiques scientifiques, ou métalangages, que l'on peut représenter de la manière suivante :

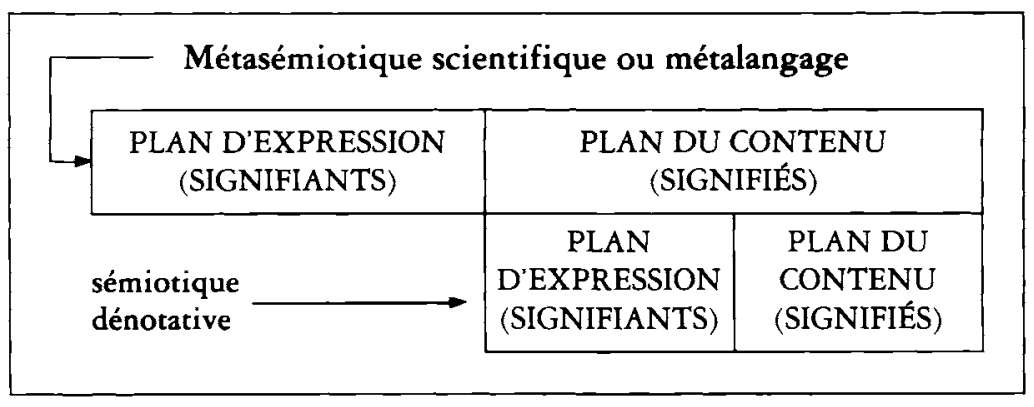


Le même schéma s'applique lorsque l'on a affaire à une sémiotique-objet non scientifique, comme c'est le plus souvent le cas en sémiologie. C'est par exemple le cas de la linguistique qui est une métasémiotique appliquée à une sémiotique dénotative non scientifique : la langue. Hjelmslev nomme justement ces sémiotiques des sémiologies.

Mais le cas inverse est aussi possible : c'est-à-dire lorsque c'est le plan de l'expression d'une sémiotique qui est une sémiotique dénotative. Le contenu, les signifiés, de cette sémiotique se rajoutent aux signifiés dénotatifs de première instance. On a alors une sémiotique connotative :

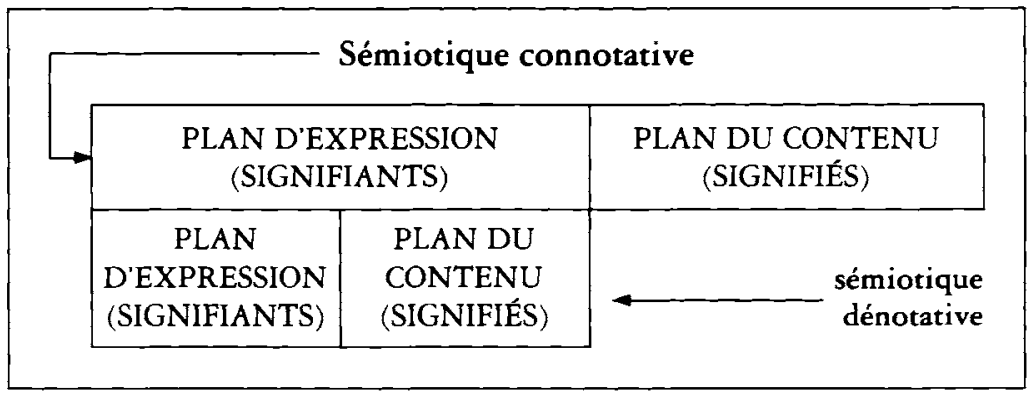

Mais ces sémiologies, métalangages et sémiotiques connotatives peuvent être prises elles-mêmes pour objet. Alors on pourra générer deux autres cas. Le premier renvoie à l'interrogation scientifique sur une sémiologie. On aura alors une métasémiologie :

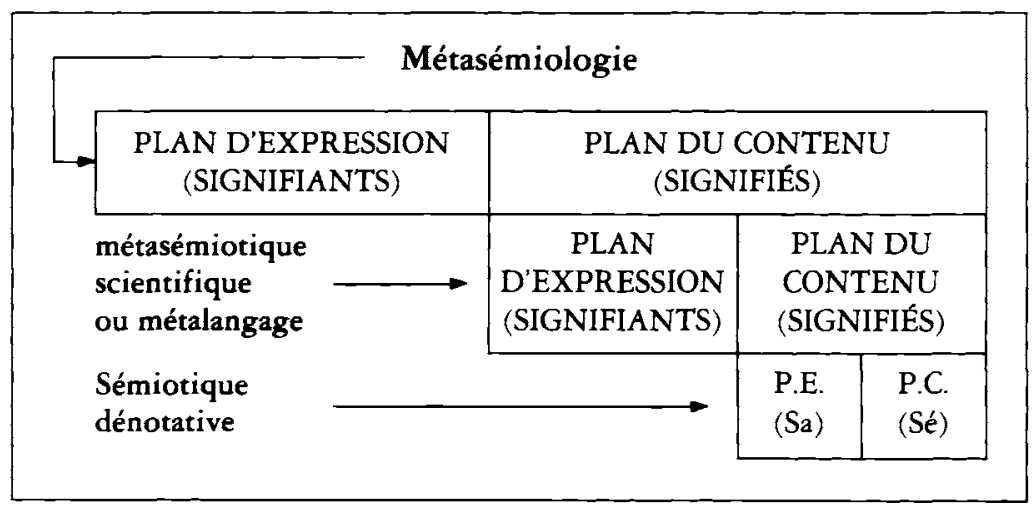


Finalement, la théorie des connotateurs relève d'une métasémiotique des sémiotiques connotatives que l'on peut représenter comme la théorie formalisée d'une sémiotique connotative dont l'objet sera le dévoilement et l'explication des systèmes de connotateurs émotifs, sociaux ou culturels. C'est là le cas qui intéresse une théorie des idéologies du point de vue hjelmslévien:

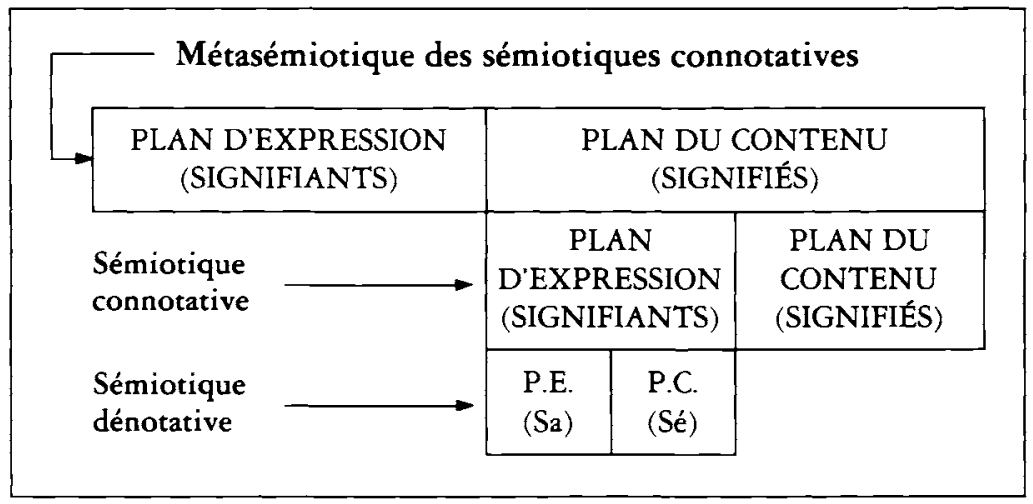

Si une sémiotique connotative est un système de signes dont le plan de l'expression est composé d'une sémiotique dénotative, la théorie de la connotation appartient à une métasémiotique dont le plan du contenu est une sémiotique connotative. Les connotateurs qui s'appliquent alors sur le premier système constituent le contenu sur lequel la métasémiotique s'appliquera, elle n'aura pas d'autre objet. Dans le cas de l'analyse textuelle, ces connotateurs pourront être des styles (forme, créativité, valeurs), des genres (parole ou écriture), des émotions ou des idiomes (régionaux, nationaux). On peut aussi représenter ce rapport algébriquement par la formule barthésienne :

$$
\text { (E.R. ((E.R.C.) R.C.)) }
$$

où E. représente le plan de l'expression;

C. est le plan du contenu;

R. la relation qui les unit en un système;

où la formule (E.R.C.) représente une sémiotique dénotative; et où la formule ((E.R.C.) R.C.) représente une sémiotique connotative. ${ }^{23}$ 
La théorie des connotations - sociales, mythologiques, géographiques ou idéologiques - est donc la théorie (l'expression et l'analyse formelle) du système des connotations. Faire la théorie d'une idéologie donnée consisterait à faire la théorie des connotateurs (signifiants) idéologiques et de leurs relations aux contenus connotés (signifiés). Ainsi s'explique Hjelmslev :

Il incombe à cette métasémiotique d'analyser les multiples sens du contenu - géographiques et historiques, politiques et sociaux, religieux, psychologiques - qui se rattachent à la nation (...), à la région (...), aux formes d'appréciation des styles, à la personnalité (...), aux mouvements, etc. ${ }^{24}$

Pour les saussuriens, c'est seulement à ce niveau qu'intervient la détermination sociale. Plus encore, le modèle de la connotation sociale qui en dérive représente pour eux la relation d'isomorphisme sociosémiotique même, de telle façon que l'étude du système des connotateurs supplée à toute mise en perspective sociologique ou historique extrinsèque.

\section{ROLAND BARTHES}

Roland Barthes, conséquent en cela avec la tradition saussurienne, en viendra à affirmer que lorsque «français » connote les messages en français, et "littérature » les œuvres littéraires :

... ces signifiés communiquent étroitement avec la culture, le savoir, l'histoire, c'est par eux, si l'on peut dire, que le monde pénètre le système ; l'idéologie serait en somme la forme (...) des signifiés de connotation, cependant que la rhétorique serait la forme des connotateurs. 25

Ainsi pourrait-on dire en paraphrasant cette pensée, la forme $\mathrm{du}$ «connotant » est rhétorique, donc fonctionne sur le mode de la persuasion, alors que la forme du «connoté » est idéologique, donc fonctionne sur le mode de la force de persuasion. "Le monde pénètre le système... » écrit Barthes, comme si le monde n'avait toujours été la source et le milieu du système ; lequel système n'est d'ailleurs jamais qu'une abstraction de l'analyste, ne l'oublions pas.

24. Prolégomènes à une théorie du langage, Paris, Minuit, 1968, 231 p. ; p. 156.

25. Éléments, Op. cit., p. 165. 
L'approche saussurienne de Barthes suscite deux critiques. D'abord elle est à la fois arbitraire et réductionniste. On voit mal comment l'idéologie, concept éminemment complexe et diversifié, pourrait se réduire à la seule " forme des signifiés de connotation », sans perdre l'essentiel de la charge conceptuelle dont il est investi. Deuxièmement, la méthode qui en découle a été incapable de rendre compte de la systématicité des idéologies et de la profondeur de l'impact de l'idéologique sur les sémiotiques naturelles. C'est Greimas lui-même qui le reconnaît à propos des Mytbologies ${ }^{26}$ qui «... ne sont que des brides connotatives et ne parviennent même pas à suggérer un système sous-jacent ». ${ }^{27}$

Certes il y a un autre Barthes; celui de la Leçon par exemple où il déclare sans ambage :

Le langage est une législation, la langue en est le code. (...)

Ainsi, par sa structure même la langue implique une relation fatale d'aliénation. Parler, et à plus forte raison discourir, ce n'est pas communiquer, comme on le répète trop souvent, c'est assujettir : toure la langue et une rection généralisée. ${ }^{28}$

La métaphore juridique est par trop évidente : le langage est un état de droit, la langue est un code législatif, les grammairiens sont des juristes et les écoles sont les commissariats de police de la langue. Ainsi le citoyen est-il aliéné, dépossédé de sa liberté de parler ; ceux qui parlent, ceux qui discourent plus encore, sont des gens de pouvoir qui assujettissent au double sens du terme: qui constituent les sujets et les dominent en même temps. La rection se dit des verbes dont le mode est déterminé grammaticalement; or si la langue est tout entière une rection (admirable métonymie), c'est qu'elle est l'établissement d'un droit sur tous les modes ou les cas du langage ; bref qu'elle est une loi universalisée. Bref, la langue se passe d'idéologie, puisqu'elle est le milieu même de toute idéologisation, son fondement en droit : "Mais la langue, comme performance de tout langage, n'est ni réactionnaire, ni progressiste ; elle est tout simplement: fasciste; car le fascisme, ce n'est pas d'empêcher de dire, c'est d'obliger à dire. ") ${ }^{29}$

26. Notons que si celles-ci sont peu systématisées, il y a néanmoins la dissertation finale, à laquelle nous référons ici, qui veut faire la théorie des mythologies

27. A.J. Greimas et J. CourTès, Sémiotique, Dictionnaire raisonné de la théorie du langage, Paris, Hachette, 1979, 423 p. ; p. 343.

28. Roland Barthes, Leçon, Paris, Seuil, 1978, 46 p. ; pp. 12 et 13.

29. Ibid., p. 14. 
La langue est bien un code, c'est le code de la législation de ce qu'il faut dire, de ce qui s'impose à dire. La langue est fasciste: totalitaire, corporatiste, nationaliste. La langue prend tout, veut régir toutes les énonciations; elle assujettit tout à ses intérêts propres, elle définit l'ordre territorial de sa rection, elle combat pour s'imposer. La langue est chauvine.

Retour du balancier, la découverte du caractère social de la langue pousse Barthes à l'autre extrémité ; les signes s'inversent. Autant la langue comme système n'était rien d'autre que la positivité de ce système, autant elle devient maintenant la forme du pouvoir totalitaire par excellence. La pensée de Barthes sur cette question est sans nuance. D'un côté comme de l'autre, il s'interdit de penser la langue comme système de significations socialement constituées, comme système marqué congénitalement au fer de la puissance de dire, et de l'énonciation d'un certain dire.

Puis dans $S / Z$, renversant la position de Hjelmslev, il écrit encore :

... la dénotation n'est pas le premier des sens, mais elle feint de l'être ; sous cette illusion, elle n'est finalement que la dernière des connotations (celle qui semble à la fois fonder et clore la lecture), le mythe supérieur grâce auquel le texte feint de retourner à la nature du langage, le langage comme nature : une phrase, quelque sens qu'elle libère, postérieurement, semble-t-il, à son énoncé, n'a-t-elle pas l'air de nous dire quelque chose de simple, de littéral, de primitif : de vrai, par rapport à quoi tout le reste (qui vient après, au-dessus) est littérature ? 30

La dénotation n'est plus le référé mais l'illusion sociale d'un référé, le mythe d'une naturalisation du référé, bref l'expression d'une idéologie particulière du rapport au monde; comme dans l'idéologie dominante, une naturalisation. Ainsi peut-on lire dans l'Idéologie allemande: [chaque nouvelle classe dominante] «... est obligée de donner à ses pensées la forme de l'universalité, de les représenter comme étant les seules raisonnables, les seules universellement valables. ${ }^{31}$

La dénotation serait cette fausse universalité du simple référé. Mais dans cette nouvelle conception barthésienne, il n'y a tout simplement plus de dénotation, puisque le dénoté n'est jamais que

30. Roland Barthes, S/Z, Paris, Seuil, 1970, pp. 15-16.

31. Op. cit., p. 46. 
la dernière des connotations. Réalisme et naturalisme de la signification primitive des énoncés se confondent dans une magnifique illusion, dans un mythe, dit Barthes, celui de la dénotation. Plutôt que sens premier, le dénoté est la connotation dernière, celle qui produit la vérité comme effet de réel. Or, dans la littérature, ce réel ne procède jamais que d'une «illusion référentielle», d'un contact sans médiation du signifiant et du référent, car "supprimé de l'énonciation réaliste à titre de signifié de dénotation, le "réel" y revient à titre de signifié de connotation. » ${ }^{32} \mathrm{Mais}$ si, en définitive, le dénoté est le dernier des connotés, alors le réel n'est jamais qu'un signifié de connotation qui crée l'illusion d'une dénotation, d'une référence au réel. Reconnaître ce fait, c'est ouvrir la porte au sens, car: «... dans l'idéologie de notre temps, la référence obsessionnelle au "concret", (...) est toujours armée comme une machine de guerre contre le sens, comme si, par une exclusion de droit, ce qui vit ne pouvait signifier, et réciproquement. ${ }^{33}$

Cette critique si exacte, si juste, adressée à l'obsession du concret, se retourne contre la conception saussurienne du dénoté : machine de guerre dans l'idéologie, contre la signification vivante, contre la vie signifiante, contre le sens.

\section{L'ÉCOLE DE PARIS}

La théorie de Greimas et de son «École de Paris » se présente comme une sémiotique générale. Parlant du projet de leur théorie, Greimas et Courtès écrivent : « fondée sur la théorie de la signification, elle vise à rendre compte de toutes les sémiotiques * (et pas seulement des langues naturelles) et à construire des modèles susceptibles de générer des discours (et non des phrases). ${ }^{34}$ Malgré les prétentions en vertu desquelles cette théorie pourrait disposer de la gestualité ou des sémiotiques naturelles, dans les faits elle ne s'applique qu'aux discours et plus particulièrement aux textes narratifs. C'est dans ce dernier domaine qu'elle a obtenu ses

32. "L'effet de réel », in Littérature et réalité, Paris, Seuil, 1982, (1968), pp. 81-90, p. 89.

33. Ibid., p. 87

34. A J. GreIMAS et J. COURTÈs, Sémiotique, Dictionnaire raisonné de la théorie du langage, Paris, Hachette, 1979. Entrée: "génératif ". p. 159. Les astérisques* renvoient à d'autres entrées du même dictionnaire. "Sémiotique » est compris dans ce passage au sens des systèmes sémiotiques de Hjelmslev. 
résultats les plus probants; les analyses appliquées aux discours juridiques, politiques ou philosophiques laissent souvent une impression d'arbitraire et, $s^{\prime}$ ils sont visés par le projet greimassien, demeurent à la périphérie de son programme de recherche. Aussi allons-nous surtout considérer cette théorie comme une sémiotique des textes narratifs; ce qu'elle est en réalité.

Le modèle de sémiotique narrative développé à l'aide du paradigme sémantique du "carré sémiotique » s'articule structurellement à l'appréhension des «isotopies » propres à un texte narratif donné. Il étaye sa compréhension du texte en décrivant les procès de sémantisation qu'il comporte comme « parcours génératifs » et systèmes de différences et d'oppositions sémantiques. Cette théorie s'intéresse peu à la matérialité et aux effets stylistiques du texte (son plan de manifestation), parce que son but est de définir une grammaire du texte. Dans cette perspective elle distingue deux niveaux d'analyse ${ }^{35}$ :

1) Le niveau de surface distingue les composantes narrative et discursive. À ce niveau, l'analyse dégage respectivement les programmes narratifs et les parcours figuratifs constitutifs des effets de sens spécifiques d'un texte.

2) Le niveau des structures profondes distingue le modèle d'opération et le modèle de relations. À ce niveau l'analyse identifie les sémèmes (combinaison des sèmes nucléaires et des classèmes ou sèmes contextuels) constitutifs des effets de sens du texte (ses valeurs sémantiques et sémiologiques), identifie les isotopies qu'ils composent et, finalement, dégage un carré sémiotique qu'elle met en œuvre et qui représente la structure générative de la signification ultime du texte.

Dans l'ensemble, l'analyse cherche à montrer comment le texte fait sens en vertu de structures sémantiques et syntaxiques de génération des énoncés propres à lui, ou au type de discours dont il est constitutif. Ainsi le texte se trouverait-il «expliqué » par ses structures.

35. Groupe d'Entrevernes, Analyse sémiotique des textes: Introduction, théorie-pratique, Lyon, Presses universitaires de Lyon, 1985, 208 p. 
L'analyse de la composante narrative comporte la description des états, comme relations spécifiques entre les sujets et les objets, et des transformations qui interviennent au cours du développement syntagmatique du texte. Ainsi, on peut dégager un programme narratif, c'est-à-dire la succession hiérarchisée des états et des transformations qui développent les relations (conjonctives et disjonctives) sujets-objets dans le texte (en définissant en outre les rôles actantiels, leur composition, leur place dans les programmes). Les programmes narratifs décrivent les successions de transformations en distinguant les phases de la manipulation, faire-faire (action sur une action), de la compétence, être du faire (modalisations : devoir, vouloir, pouvoir, savoir), de la performance, faireêtre (définition des valeurs) et de la sanction, être de l'être (véridiction de l'être et du paraître, cognition). Un programme narratif définit le résultat d'ensemble des diverses transformations d'état que décrit le texte (acquisition ou perte d'objets modaux, cognitifs ou valeurs). Un texte peut évidemment comporter divers programmes hiérarchisés. C'est ce qui constitue le cadre de la pragmatique greimassienne.

Dans la composante discursive, on définit les figures spécifiques qui investissent le rôle du sujet. Le premier aspect de l'analyse figurative consiste à retrouver les lexèmes divers qui informent le texte, tant comme noyaux stables de signification que comme "parcours sémémiques» (divers sens liés au noyau). Les figures d'un discours forment un réseau lexématique dont les occurrences dans le déroulement du discours constituent un parcours figuratif. Les redondances de ce parcours nous indiquent la configuration discursive des thèmes (rôles thématiques des acteurs) et des motifs du texte. Les parcours sont les unités de ce «dictionnaire » discursif propre à un texte ou à un corpus donné qui sont réalisées dans les programmes narratifs.

L'analyse des structures profondes décroche de l'axe syntagmatique pour retrouver les ressorts sous-jacents de la performativité sémantique du texte. On a vu que les figures lexématiques peuvent être comprises comme divers parcours sémémiques à partir d'un noyau stable. Ces sémèmes sont des compositions d'unités minimales de signification : les sèmes. Les sèmes sont, comme les lexèmes et les sémèmes, distingués entre eux non substantiellement mais par 
différenciations. Ils se composent pour former les sémèmes. Par exemple, on pourrait (sans en épuiser le sens) établir que :

$$
\begin{aligned}
& \text { automobile }=/ \text { véhicule } /+/ \text { quatre roues } /+/ \text { motorisé } / \\
& + \text { /passagers/ + /léger/ } \\
& \text { autobus }=/ \text { véhicule } /+/ \text { quatre roues } /+/ \text { motorisé } / \\
& + \text { /passagers/ + /lourd/ } \\
& \text { camion }=/ \text { véhicule } /+/ \text { quatre roues } /+/ \text { motorisé } / \\
& + \text { /choses/ + /lourd/ }
\end{aligned}
$$

Dans cet exemple, le noyau sémémique commun est: /véhicule/ + /quatre roues/ + /motorisé/. Et l'opposition sémique qui différencie le premier du second est /léger/ vs /lourd/ et le second du troisième est / passagers/ vs/choses/. Une analyse similaire peut être appliquée aux configurations discursives qui, composées de divers parcours figuratifs, sont aussi assimilables à des compositions de sèmes. On distinguera les sèmes nucléaires (qui appartiennent à une figure lexématique ou discursive en propre) qui définissent le niveau sémiologique, et les classèmes (qui appartiennent au contexte phrastique et discursif d'une figure) qui définissent son niveau sémantique.

La redondance et la régularité des sèmes nucléaires forment des isotopies sémiologiques (ou grammaticales) et la redondance et la régularité des classèmes forment des isotopies sémantiques par lesquelles la cohérence des figures d'un texte est assurée. En particulier, les isotopies permettent la «désambiguïsation 》 des expressions, ou même des grandes unités du discours. Par exemple, les oppositions sémiques distinctives sont isotopes, de même que le carré sémiotique. Cette analyse peut, selon l'École de Paris, être étendue aux parcours figuratifs : l'isotopie sémiologique assurant la permanence des parcours, et l'isotopie sémantique assurant la cohérence d'ensemble de tous les parcours du texte dans une unité de sens.

Le carré sémiotique est un modèle formel représentant la structure ultime de signification d'un texte. Il se construit à partir d'une structure élémentaire oppositive et hiérarchique. Voici un exemple d'une telle structure: 


\section{/ignorance/ vs /connaissance/ \\ $/ 1$ \\ /technique/ vs / scientifique/ \\ $/ 1$ \\ /pure/ vs /appliquée/}

L'axe paradigmatique des figures est généralement organisé par de telles structures oppositives. La thèse forte de Greimas consiste à affirmer que pour être sensé, tout texte doit être organisé par des relations obéissant à la forme générale abstraite du carré sémiotique, dont voici une présentation synthétique ${ }^{36}$ :

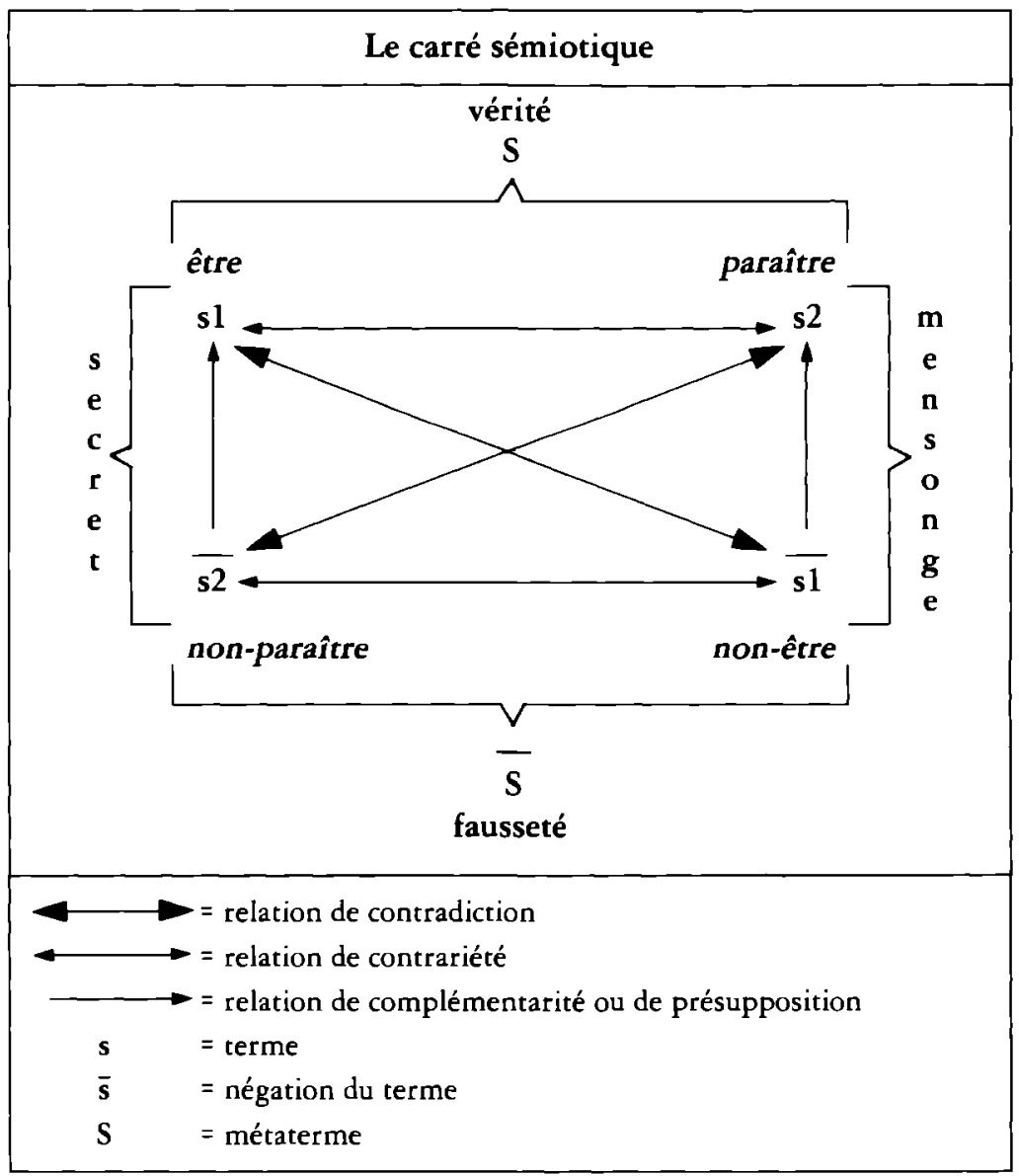


Le carré sémiotique est une représentation des relations sémantiques de contrariété, de contradiction et de complémentarité entre des termes, des figures, des modalités ou des sèmes. Le carré sémiotique forme l'isotopie fondamentale d'un texte. Voici les diverses relations qui y sont représentées :

- relations biérarchiques: les métatermes subsument les termes;

- relations de contrariété (et subcontrariété) : un rapport d'incompatibilité et de double présupposition (ou de double complémentarité) unit les termes ;

- relations de contradiction: les termes se nient mutuellement ;

- relations de présupposition, de complémentarité ou d'implication narrative : les termes négatifs sont présupposés par leurs compléments, asserter la négation de $\mathbf{s} 1$, c'est impliquer s2 ;

- relation contradictoire des métatermes : deux relations de contrariété contractent une relation de contradiction à un niveau hiérarchique supérieur (vérité et fausseté sont contradictoires);

- relation contraire des métatermes: deux relations complémentaires contractent une relation de contrariété (secret et mensonge sont contraires).

L'analyse sémiotique d'un texte consiste donc à construire la (ou les) structure(s) profonde(s) qui le caractérise(nt), c'est-à-dire à extraire le couple de contraires qui est au principe de sa signification. À une seconde étape le carré sémiotique est mis en mouvement par l'identification des opérations de transformations sémantiques profondes qui activent le texte : négation sur les axes de la contradiction (axes des schémas positifs, $s 1-\sim s 1$, et négatif, s2- s2); sélection sur les axes de la présupposition (axe de la déixis négative, $\sim \mathrm{s} 1-\mathrm{s} 2$, et de la déixis positive, $\sim \mathrm{s} 2-\mathrm{s} 1$ ). Ainsi, on aura les transformations suivantes : une première opération de négation effectuera le passage $s 1 \rightarrow \sim s 1$; une seconde opération de sélection fera passer $\sim \mathrm{s} 1 \rightarrow \mathrm{s} 2$; une troisième de négation effectuera

36. Synthèse des schémas suivants : Dictionnaire, op. cit., pp. 31 et 32 ; Analyse.., op. cit., pp. 132 et 138 . L'exemple paradigmatique est de Greimas et Courtès, il représente le schéma de véridiction de la modalisation des énoncés d'états. 
le passage $s 2 \rightarrow \sim s 2$; et finalement une quatrième opération de sélection fera passer $\sim \mathrm{s} 2 \rightarrow \mathrm{s} 1$. Le tout décrit un circuit de transformation.

$\mathrm{Si}$, au niveau de surface, la composante narrative est une espèce de grammaire du texte, alors les programmes narratifs sont présumés prendre en charge la réalisation des opérations de transformation du niveau profond; tout comme le dictionnaire de réseaux figuratifs réalise les relations du niveau profond. C'est en ce sens qu'on parle de générativité. Le niveau profond est une logique qui se réalise dans le récit de surface. Le carré sémiotique représente la forme du contenu d'un texte et vérifie sa cohérence. De plus, les sèmes inscrits aux quatre coins du carré sémiotique se conjuguent avec d'autres sèmes, de manière à ce que puissent être générés les divers sémèmes du réseau figuratif, ou les diverses combinatoires de la composante discursive. Bref, le carré sémiotique est l'aboutissement de cette théorie et le modèle qui prétend représenter la sémantique et la dynamique de production de sens des textes et des discours. Ainsi se termine notre succincte présentation de cette vaste théorie aux multiples applications.

Nous allons commencer notre critique par quelques commentaires sur la véritable nature du carré sémiotique. L'intuition fondamentale du carré sémiotique, et jusqu'à ses structures les plus caractéristiques, se trouve entièrement dans la logique aristotélicienne ; seul le champ d'application diffère. La logique contemporaine a formalisé les analyses d'Aristote sur les propositions sous la forme générale du carré logique. Elle a fait de même à propos des modalités aristotéliciennes sous la forme du carré des modalités (qui est aussi à la base de la logique déontique) ${ }^{37}$. Mais la logique contemporaine n'a pas beaucoup porté attention aux oppositions catégorielles chez Aristote. Le carré sémiotique est une extension de cette logique catégorielle aristotélicienne.

37. S. RoBert, La logique, son histoire, ses fondements. Longueuil, Le Préambule, Pp. 25 45 ; Louis VAX, Logique, op. cit., PP. 89-92 et 109-115; Émile BRÉHIER, Histoire de la philosophie, tome I, Antiquité et moyen-âge, Paris, P.U.F., 1981, Pp. 152-166; ARISTOTE, Organon, I-Catégories, II-De l'interprétation, Paris, Vrin, 1977, traduction et commentaires de J. Tricot, 153 p., pp. 42-68, 104-114 et 137-144. 
La théorie de Greimas n'est pas une logique propositionnelle ${ }^{38}$; aussi allons-nous en rester au plan catégoriel. Aristore étudie quatre types d'oppositions entre les termes : les relatifs, les contraires, la possession et la privation, et la contradiction ${ }^{39}$. Il explique que certaines catégories ne peuvent être pensées dans un schéma de contrariété, par exemple certains relatifs: «Cependant tous les relatifs n'ont pas de contraires: au double n'est opposé aucun contraire, ni au triple, ni à aucune terme de ce genre. ${ }^{40}$; ou certaines qualités «... le rouge, le jaune et les couleurs de cette sorte n'ont pas de contraires, bien que ce soient des qualités. ${ }^{41} \mathrm{Ce}$ point doit être retenu car Greimas n'en tient aucun compte pour ne conserver que les schémas oppositifs. En tout état de cause, Aristore insiste sur l'importance de déterminer le genre dont les oppositifs font parties ou le sujet auquel ils se rapportent ${ }^{42}$.

A ristote distingue l'opposition des contraires et l'opposition des contradictoires ( ce qui est opposé comme l'affirmation et la négation ${ }^{43}$ ). Les contradictoires s'opposent selon la vérité et la fausseté. « Socrate est malade » et «Socrate n'est pas malade » sont des affirmations contradictoires et exclusives l'une de l'autre ; cela indépendamment de l'existence ou de l'inexistence de Socrate. Rien de tel avec les contraires : "Socrate est malade » et «Socrate est bien portant » sont contraires. Ces propositions peuvent cependant être sans objet; ainsi la fausseté de l'un n'implique pas nécessairement la vérité de l'autre. On voit qu'avec ce jeu d'oppositions / malade/ vs /bien portant/ et /malade/ vs / non-malade/ (qui implique /bien portant/vs / non bien portant/) nous avons toutes les oppositions catégorielles et les relations du carré sémiotique. Aristote donne comme exemple de contraires «blanc» et

38. Ce choix est étonnant en ce sens que le discours a plus d'affinité avec la proposition qu'avec les simples catégories; mais on sait depuis longtemps que la logique propositionnelle est trop restrictive pour rendre compte du langage ordinaire, des phrases et $a$ fortiori des unités transphrastiques. Greimas régresse en quelque sorte vers un modèle logique encore plus élémentaire, la logique des catégories, et done qui apparaît intuitivement moins apre à rendre compre du complexe. Ce choix ne s'explique que par sa recherche d'unités minimales de signification.

39. Op. cit., Catégories, pp. 29-68.

40. Ibid., p. 31

41. Ibid., P. 50 .

42. Ibid, p. 66-68.

43. Ibid., p. 64 
«noir», et comme exemple de contradictoires «homme» et "non-homme». Comme on le constate, les oppositions du carré sémiotique sont congruentes avec cette théorie si et seulement si les contraires s'opposent en effet et les contradictoires s'excluent.

L'analyse des propositions et de ce que nous nommons maintenant le carré logique fait partie pour Aristote de l'analyse d'une certaine sorte de discours (à l'exclusion des discours qui ne relèvent pas directement du critère de vérité, relégués aux domaines de la rhétorique et de la poétique). Ainsi les règles qui régissent la contrariété, la contradiction et la subalternation ne sont-elles applicables qu'aux catégories et propositions logiques. C'est là une autre nuance que Greimas passe sous silence dans sa reprise d'Aristote. On doit noter également que Greimas utilise les foncteurs de la logique des propositions (disjonction, conjonction, négation) sans tenir aucun compte des lois logiques qui en régissent l'usage, puisqu'il ne les utilise qu'en un sens descriptif.

Comme on le voit aisément la théorie du carré sémiotique est fortement dépendante de la logique aristotélicienne, bien qu'elle n'en retienne qu'une partie. En particulier, elle passe sous silence la théorie des syllogismes qui est pourtant l'aboutissement naturel de ce que nous venons de voir. Par contre, il est une règle qu'elle respecte scrupuleusement: la loi du tiers-exclu. En vertu de cet axiome, il est exclu d'attribuer à un terme ou à une proposition une valeur différente de celles de vérité et de fausseté. Si on a $A$ vrai, alors $\tilde{A}$ est nécessairement faux. C'est là un axiome qui est quelquefois contesté dans les logiques formelles contemporaines ; qu'on pense simplement aux logiques plurivalentes et probabilistes qui admettent une, plusieurs ou même une infinité de valeurs intermédiaires entre le vrai et le faux. En particulier certains systèmes logiques admettent la valeur «indéterminé ». Greimas ne tient aucun compte de ces développements qui permettent aux logiciens de former des énoncés formels plus proches des formes discursives ordinaires. Il s'en tient à une opposition binaire classique que plusieurs logiciens et philosophes du langage jugent inappropriée pour rendre compte du langage ordinaire : ce que Greimas prétend pourtant faire. Plus encore, il prétend que la structure profonde qui régit la signification de tout discours est binaire et fondée sur l'axiome du tiers-exclu. Voilà une thèse excessivement générale et donc extrêmement vulnérable sur le plan empirique, sujette à une 
réfutation facile; or cette thèse est au cœur même de sa théorie (relayant en cela le modèle saussuro-hjelmslévien).

Le Groupe d'Entrevernes écrit à ce propos de la binarité du modèle :

Ce choix théorique de la binarité peut paraître gênant. Toutefois, il convient d'admettre que cette articulation binaire ne porte pas sur les "choses du monde », ni même sur le "sens » d'un texte. Ce qui est mis en relation binaire, ce sont les unités élémentaires construites de la signification. (...)

La binarité est donc bien à concevoir comme une règle de construction des unités de sens.

À ce titre, elle tire sa justification du caractère opératoire du modèle qu'elle permet de mettre en place. ${ }^{44}$

Admettons que la binarité n'ait aucune signification métaphysique ici et ne prétende pas non plus rendre compte du sens immanent des textes. Admettons qu'il s'agisse d'un outil purement abstrait (comme le code binaire des ordinateurs) dont les éléments sont de simples abstractions d'une règle de représentation qui n'appartient qu'à la syntaxe de la théorie, et non à la syntaxe des textes. Supposons enfin que cette règle ne se juge qu'à ses fruits. Il n'en demeure pas moins que ces oppositions binaires ne jouent pas seulement un rôle dans la théorie, mais aussi dans la description même des structures de signification des textes. Ainsi ne peut-on le rabattre exclusivement au niveau des caractéristiques du formalisme scientifique. Ils admettent eux-mêmes qu'au-delà cela ne fait pas sens. Mais justement, le modèle greimassien va au-delà et postule une structuration binaire des axes paradigmatiques différenciateurs des modalités, des actions, des actants, des parcours figuratifs et des programmes narratifs, des structures discursives et des structures profondes de génération de la signification. Bref, le binarisme est partout dans la description, dans l'explication et dans la générativité. On ne peut en faire un simple outil et la comparaison avec les circuits informatiques est totalement «irrelevant». C'est donc bien ce postulat dont il faut vérifier la validité. 
Dans le même esprit, J.C. Coquet écrit que la fonction polémique du discours est parfaitement représentée dans le modèle greimassien. Il compare cette "pragmatique» du texte à l'analyse de «la relation intention-effet » ou du «rapport action-réaction» chez Peirce, et citant Gérard Deledalle il souligne que toute secondéité comporte un élément de lutte ${ }^{45}$. Le sens de la déformation qu'il fait subir à la théorie peircienne est singulier, puisque précisément Peirce, et Deledalle à sa suite, sont de farouches adversaires de la conception duelle de la signification. Certes, Peirce analyse la secondéité, mais la vérité de la secondéité et le mouvement authentique de la signification se situent pour lui au plan irréductible de la tiercéité, de l'interprétant : la secondéité n'est qu'un intermédiaire obligé, celui de la réalité singulière, de l'existence brute dont les principes se trouvent ailleurs. Par son interprétation tronquée, Coquet avoue que l'analyse de l'École de Paris est congénitalement limitée à l'étude des dyades et donc n'accède jamais à la signification, puisqu'elle se cantonne dans l'élément du sens : des tokens. Exclure le tiers, c'est exclure l'interprétant et donc toute signification.

La sémiotique greimassienne se veut un modèle de génération de la signification discursive, mais elle impose a priori aux textes des principes et des règles qui leur sont étrangers. Toutes les relations et les transformations présumées sont duelles. Toutes les oppositions sémantiques sont des dyades. Toutes les hiérarchies reposent sur la reconduction des dualités. Toutes les assignations de valeurs excluent le tiers. Bref, il s'agit d'une sémiotique aristotélicienne et, qui plus est, tronquée. Ses principes de logique sont inadéquats pour représenter les subtilités des discours qui n'obéissent ni au principe binaire, ni au tiers-exclu (à la seule exception des formulations strictement mathématiques ou logico-formelles).

Greimas mésinterprète Aristote puisqu'il confond la relation de contrariété et la simple différenciation. Ses exemples abstraits obéissent à la définition de la contrariété, mais ses analyses concrètes substituent forcément aux véritables relations de contrariété de simples différenciations de termes. En effet, les oppositions de termes ou de sèmes ne sont pas substantielles mais dépendent des valeurs différencielles qu'ils prennent dans la langue sur l'axe

45. J.C. CoQUET, «L'École de Paris», Sémiotique..., op. cit., p. 29. 
paradigmatique et aussi selon les contextes. À la limite, ces oppositions relèvent d'un arbitraire linguistique et culturel. Le problème principal de cette sémiotique est le suivant : comment sait-on que tous les termes, tous les sèmes et toutes les figures et parcours obéissent à cette règle de différenciation binaire ? Aristote lui-même mentionne des cas où ce n'est pas possible d'opérer une telle différenciation. Certes, on peut forcer le discours pour le contraindre à rentrer dans une structure binaire: mais alors ce ne sont plus les caractéristiques du texte que nous étudions, mais celles de nos postulats; nous ne sommes plus dans la science empirique, mais dans un pur formalisme, ce qu'évidemment le sémioticien veut éviter. Par exemple, les sentiments rentrent très mal dans un schéma binaire: doit-on alors bannir les sentiments non binaires de la génèse du sens des textes? En outre, il existe des catégories, des termes et des structures non oppositives: par exemple les structures analogiques de la communication. Devrat-on refuser d'en rendre compte?

L'analyse de la structure de surface peut être utile mais n'explique rien: elle ne fait que décrire de manière très complexe ce que le lecteur moyen comprend aisément. Les efforts pour dynamiser le modèle ne font qu'accentuer ses défauts mécanicistes, puisque tout mouvement n'est que le passage par la négation ou l'inclusion présuppositionnelle. Cela ne surprend nullement puisque tout dynamisme authentique exige l'intervention d'un tiers qui décentre le mouvement et le fait accéder à un autre univers de signification. Au contraire, les structures duelles, même indéfiniment redoublées, ne peuvent que « tourner en rond "et revenir finalement à leur point de départ, puisqu'elles ne sont que les figures complexifiées du mouvement d'aller-retour, d'affirmation-négation. Les prétentions du carré sémiotique à représenter toute structure de signification discursive ne sont pas exagérées, elles sont dérisoires ! C'est un peu comme si on tentait de rendre compte de tout énoncé en se servant exclusivement du calcul des propositions : on n'aurait ni modalité, ni quantification, ni indétermination, ni même narrativité. Or le carré sémiotique prétend faire plus encore : réduire les complexes structures de la signification discursive à des oppositions catégorielles qui sont encore plus simples et plus élémentaires que celles de la plus banale syllogistique, pourtant universellement reconnue comme insuffisante. 
D'ailleurs, cette théorie n'a de grammaire ou de syntaxe que le nom. Non seulement sa sémantique est pauvre, et sa pragmatique inadéquate, mais en plus tout ce qu'elle décrit sous ce nom n'est qu'une imitation pauvre et rigide d'une véritable syntaxe. Au plan épistémologique, il est notable que malgré ses prétentions explicatives, la théorie de Greimas ne produise jamais que des descriptions de textes, en général très peu économiques, voire carrément subjectives, cela malgré tout l'appareillage conceptuel mis en œuvre. Le choix des catégories oppositionnelles est toujours discutable ; ainsi la méthode greimasienne n'est-elle qu'une forme d'interprétation complexe, ordonnée autour d'un schéma inapproprié à la plupart des cas.

Au mieux, le carré sémiotique peut-il avoir une utilité descriptive provisoire et limitée aux structures de signification binaires avérées. Son attachement à une logique aristotélicienne affaiblie le rend inapte à appréhender la plus simple des significations non propositionnelles.

Maintenant examinons le statut qui est réservé à la sociosémiotique dans cette «théorie ». Greimas et Courtès écrivent :

L'universalité de la culture et les spécificités culturelles constituent une des visées de la théorie sémiotique qui cherche à les atteindre et à les analyser systématiquement à travers la diversité des sémiotiques saisissables comme des axiologies * ou comme des idéologies *, et définissables comme des modèles d'action * et de manipulation *. À la sociosémiotique (...) serait réservé le vaste domaine des connotations * sociales... ${ }^{46}$

«Idéologie» est opposé à «axiologie»: le dernier terme représente une organisation systématique de valeurs, alors que le premier représente l'organisation actuelle (de surface) et l'actualisation de la quête de ces valeurs dans le discours (comme structure actantielle). Les actions et les manipulations ici décrites concernent les rapports discursifs définissant des actes, soit de l'humain sur des choses (action), soit de l'humain sur l'humain (manipulation); mais toujours dans le discours.

46. A.J. Greimas et J. Courtès, lbid., entrée : «Sociosémiotique », p. 356. 
Selon cette école, à la seule exception de Chabrol qui préconise une approche plus empiriste ${ }^{47}$, la sociosémiotique, dont la sociolinguistique est une branche particulière, ne doit pas faire appel à des facteurs extra-sémiotiques: de leur point de vue, le modèle dénotation/connotation suffit à établir l'ensemble des rapports, d'ailleurs immanents au discours, entre les signifiants et les signifiés sociaux. Ainsi :

Par approche socio-linguistique il faut entendre la description des différenciations et des articulations significatives des sociétés bumaines, description fondée sur l'enregistrement des écarts significatifs au niveau des langues naturelles. C'est dans la mesure seulement où l'on peut enregistrer des écarts, grands ou petits, au niveau des langues naturelles, considérées comme signifiant social, qu'on peut leur attribuer un signifié social, constitué de l'ensemble des connotations sociales. Autrement dit, les procédures de découverte et de description sociolinguistiques doivent être situées sur un plan bomogène et unique, elles ne consistent pas dans l'établissement de comparaisons entre catégories linguistiques d'un côté, et catégories sociologiques de l'autre. ${ }^{48}$

Greimas reconduit l'erreur formaliste de Saussure (critiquée excellement par Bakhtine) : en effet, il suppose que le social peut se saisir dans le discours, sans saisir aussi comment les contextes sociaux interviennent sur sa substance même, bref en ne retenant du social que ses marques connotées, sa pure syntaxe. Néanmoins, il faut reconnaître qu'il affirme la possibilité d'engager l'étude d'un «langage de connotation sociale». Selon lui, la sociosémiotique devrait mettre en relation les pratiques linguistiques avec d'autres

47. Claude Chabrol, "Pour une psycho-socio-sémiotique ". Dans J.C. Coquet et al, Sémiotique, l'École de Paris, Paris, Hachette, 1982, 207 p., pp. 173-198.

Chabrol écrit à propos du projet greimassien : «Nous acceptons pour notre part ces objectifs comme un programme à long terme mais dans l'immédiat il nous paraît peutêtre trop ambitieux. (...) Nous croyons à la nécessité provisoire d'adopter dans un premier temps des démarches inductives, empiriques et interdisciplinaires (done hétérogènes du point de vue théorique) pour renouveller ces recherches (...) a) des enquêtes psycho-socio-sémiotiques descriptives et comparatives... b) des études microdiscursives qui privilégieront l'analyse discursive interlocutoire... »; ; p. 176.

Notre démarche est tout à fait en accord avec ce point de vue; cependant il contredit l'entreprise greimassienne elle-même, et ouvre la porte à une refonte théorique, puisque ces excursions ne pourront que conclure sur la nécessité de la conjonction de perspectives disciplinaires diverses. Une telle conjonction construit un nouvel objet désormais irréductible à la seule sémiotique, surtout saussurienne !

48. A J. Greimas, Sémiotique et sciences sociales, Paris, Seuil, 1976, 216 p.; p. 67. Nous soulignons. 
pratiques signifiantes. $\grave{A}$ ce propos, il donne en exemple les «modes de vie »; remarquons l'erreur sociologique au passage : un mode de vie n'est pas un texte, ses structures symboliques sont autres, impossibles à réduire au simple discursif. Mais cette perspective est intratextuelle; donc on vise ici non le mode de vie, mais le discours sur le mode de vie. L'exclusion des facteurs extrasémiotiques se pose à nouveau mais différemment et rend impossible une authentique approche sémiotique pour ne produire qu'une linguistique discursive comparative des textes.

Si Greimas doute, à juste titre, de la pertinence des facteurs sociaux identifiés par la sociologie fonctionnaliste dans l'explication sémiotique, il a tort de ne considérer le procès de l'énonciation par des «sujets compétents » que du strict point de vue de l'énoncé. Si l'analyse du discours permet de saisir l'«épistémologie mythique », l'«ontologie culturelle» d'une culture donnée, comme de dresser une « taxinomie de langages sociaux», on voit mal comment, sans recourir aux concepts et aux méthodes particulières des sciences sociales, on pourrait appréhender la signification sociale et politique de ces discours. C'est d'autant plus vrai lorsqu'on se propose d'étudier «ces nouvelles formes sémiotiques [qui] se développent [et] qui tendent à raffermir la cohésion sociale ébranlée ${ }^{49}$, la sociolittérature ou les «sémiotiques sociospectaculaires ». Outre la démarche, prudente mais constitutivement restreinte, de Greimas, ce qui est en cause ici c'est l'objet même de la sémiotique : doit-on limiter l'objet de la sémiotique au point de ne plus reconnaître de pertinence qu'aux discours narratifs et, qui plus est, seulement d'un point de vue internaliste? Le social n'est-il pas présent dès l'origine, aussi bien dans les sémiotiques dénotatives que dans les sémiotiques connotatives?

Si la sémiotique peircienne, qui est beaucoup plus générale, peut intégrer ces éléments, c'est précisément parce qu'elle est une sémiotique pragmatiste et dialectique, donc totalement opposée aux explications par des dyades et les formes symboliques abstraites de leurs contextes. Leur tendance à figer les oppositions sémantiques du carré sémiotique, le dyadisme fondamental de leur approche de la dynamique du sens et surtout leur vision étriquée des phénomènes pragmatiques - où la structure narrative surdétermine toute

49. Dictıonnaire..., op. cit., p. 357 
autre forme de signifiance - rend l'ensemble de leur approche incompatible avec l'élaboration d'une sociosémiotique telle que nous la concevons. Dans le cadre de la sémiotique peircienne, leur analyse ne représente qu'une simple réplique inauthentique de la tiercéité sous le mode imparfait de la dyade : pensée simplement relationnelle, donc figée. En conséquence, elle n'est d'aucune pertinence pour une théorie de l'idéologie.

\section{UNE SÉMIOLOGIE SOCIALE SAUSSURIENNE}

Il faut reconnaître que ces sémiologies réductrices ne sont pas les seules qui peuvent être inspirées par la pensée de Saussure; déjà nous avons vu le Barthes de la Leçon. Mais les exceptions auraient ici la faculté de nous montrer comment il est difficile de penser le social à l'intérieur de la problématique dichotomiste et statique saussurienne puisqu'à chaque avancée sérieuse en ce sens, c'est tout le modèle qui se trouve mis en difficulté.

Prenons à témoin de cette démarche dans ce qu'elle a de plus fécond le travail de Louis-Jean Calvet. ${ }^{50}$ Ce dernier critique la conception dominante du saussurisme, selon laquelle la connotation se définit comme une seconde signification qui vient se rajouter au signe, dans l'usage, sous la pression du contexte idéologique de la communication. Il remarque que la connotation constitue une doxa qui conforte l'idéologie dominante, mais qu'elle «n'est pas statique, elle entretient au contraire avec la dénotation un rapport dynamique, évolutif ". ${ }^{51}$ Dans le cas de la colonisation, par exemple, même la dénotation est affectée par le racisme : «... la connotation passe peu à peu dans la dénotation $\gg .{ }^{52}$ Mais encore :

Et nous avons là une importante direction de travail qui partirait du postulat que l'évolution sémantique consiste en un constant va-et-vient entre la connotation et la dénotation. Ce qui, de notre point de vue (...), revêt une importance extrême, car nous trouvons là la démonstration que la connotation, lieu privilégié où se manifeste l'idéologie, n'est pas surajoutée à la langue, fait de parole, d'usage de l'instrument, mais qu'au contraire elle en est constitutive. Ce qui, du même coup, vient

\footnotetext{
50. Nous nous limitons ici à Pour et contre Saussure. Vers une linguistique sociale, Paris, Payor, 1975, $152 \mathrm{P}$.

51. Ibid., p. 127.

52. Ibid, p. 128.
} 
conforter tout ce que j'ai jusqu'ici proposé quant au refus de l'abstraction du code par rapport à la pratique sociale : c'est dans cette pratique, et sous l'influence parmi d'autres facteurs, de l'idéologie, que ce fait, à chaque instant, la langue. ${ }^{53}$

Testons avec un exemple: le mot/sauvage/. Le premier dénoté de sauvage est : qui vit à l'état de nature. Au sens humain (connoté), on dira : est sauvage qui vir à l'état de nature, c'est alors un synonyme de "primitif ». Graduellement (connoté de second degré), ce qualificatif s'appliquera aux personnes farouches, insociables. Mais le connoté, appliqué systématiquement aux améridiens, finit (au Canada) par se charger de toutes les conceptions racistes négatives que l'on applique aux "sauvages»; ils sont paresseux, destructeurs, irrespectueux. Le terme / sauvage/ finalement dénote les dites qualités ; un sauvage est un être invivable. Dans le langage courant, le connoté a envahi le dénoté (bien que le dictionnaire maintienne une hiérarchie anachronique); le véritable dénoté de /sauvage/ est désormais ce caractère insociable, brutal, «c'est un véritable sauvage ». La pratique sociale, idéologique, a modulé la langue. Un effet de bougé important affecte la conception saussurienne des rapports entre la dénotation et la connotation.

Les questions sociales et psychologiques que pose la phrase t'es pas un mec, t'es une vraie gonzesse dépasse de très loin le cadre de la sociolinguistique ou de la psycholinguistique qui ont aujourd'hui cours. C'est cela qui paraît fondamental, et c'est à cela qu'il faut s'arrêter : le rapport de force, la violence, la pulsion, l'idéologie. ${ }^{54}$

L'idéologie - sexiste, machiste - qui se manifeste dans l'expression bien parisienne, / $t$ 'es pas un mec, t'es une vraie gonzesse/, l'allusion ténue, ambiguë, au désir sexuel, est, bien audelà d'une simple connotation du dénoté, la marque véritable dans la langue, dans l'expression, de déterminants psychologiques et sociaux: la force, le désir. On ne peut repousser le trouble aux frontières de la langue, car une passion y est toujours investie.

Kerbrat-Orrechionni prend également ses distances avec la théorie saussurienne, et surtout avec son interprétation hjelmslévienne, dans une excellente revue critique des diverses acceptions 
du concept de connotation. 55 Tout d'abord elle cherche à montrer l'importance du concept. Discutant à propos de la «classique théorie du signe ", elle écrit :

Pour moi, les séductions et les mérites qui s'attachent à la connotation tiennent essentiellement à ceci :

- d'une part, elle entraîne inéluctablement la réflexion linguistique vers les champs contigus du poétique, du psychologique, du sociologique, et de l'idéologique;

- d'autre part, elle ouvre sur la problématique de la «signifiance »: (...) pervertissant l'égalité dénotative, la connotation démontre, à l'évidence que les mécanismes de production du sens sont infiniment plus complexes, (...) plus subtils, plus indirects aussi parfois... 56

Bref s'interroger sur la connotation revient à s'interroger sur tous les aspects de la langue qui entraîne la linguistique vers les champs limitrophes des autres sciences humaines, et à explorer, au-delà des mécanismes de la signification, ceux de la signifiance.

Voilà qui est fort prometteur, mais la question qu'il faut poser est celle-ci : le concept de connotation est-il un instrument théorique approprié pour traiter d'une série de questions fort complexes et diversifiées, en même temps que de rendre compte des mécanismes fondamentaux de la signifiance? Les distinctions entre le signifiant et le signifié de connotation, entre la forme et la substance des connotateurs, entre les types de connotation, forment-elles un réseau de distinctions propres à couvrir adéquatement le champ des objets visés? Notre réponse est simplement : «non». Non, car, outre les arguments déjà élaborés, une objection nous semble s'imposer : la diversification et l'ampleur même des objets visés, le caractère exclusivement classificatoire des théories montrent que le concept de connotation est ni plus ni moins que la «vertu dormitive » des sémiologues. Cela est social puisque c'est connoté socialement; cela est connoté puisque le social s'y fait sentir! «Connotation » n'est que le nom des questions exclues arbitrairement de la linguistique par le saussurisme; les connotateurs sont un répertoire d'anomalies que la théorie saussurienne est incapable de réduire. La connotation est le roc de signifiance sur lequel la nef saussurienne vient se briser.

55. Catherine Kerbrat-Orecchionni, La connotation, Lyon, Presses Universitaires de Lyon, 1977, $256 \mathrm{p}$.

56. Ibid., Pp. $7-8$. 
À propos des sémiotiques connotatives de Hjelmslev, KerbratOrrechionni écrit justement :

La symétrie est séduisante, mais à notre avis illusoire. Cette représentation, trop rapidement acceprée par la plupart des linguistes, ne rend compte adéquatement, ni de la nature du métalangage (un discours qui parle du langage n'est pas pour autant un "langage dont le contenu est déjà un langage »), ni de la totalité des mécanismes connotatifs, dont le support est à la fois plus autonome et plus diversifié que ne le laisse supposer le modèle hjelmslévien. ${ }^{57}$

Si cette critique est juste, alors c'est le noyau même de la théorie hjelmslévienne qui est gravement atteint. En effet, toute la conceptualité de Hjelmslev repose sur cette double possibilité de réduire la connotation à n'être que le second contenu d'un langage dénotatif, et de rendre compte scientifiquement de ces phénomènes par des métalangages purement formels. Si l'on admet :

1) que les mécanismes connotatifs sont plus autonomes visà-vis des langages dénotatifs qu'on ne le supposait ;

2) qu'ils sont plus diversifiés;

3) et que les métalangages sont autres choses que de simples systèmes d'expressions appliqués à d'autres langages;

alors on ne peut se contenter d'un simple réaménagement et d'un élargissement de la théorie de la connotation pour rendre compte des objets visés, comme Kerbrat-Orrechionni le suggère; il est plus économique pour la théorie d'abandonner un modèle si défectueux.

Plus encore :

... croire que « tout connotateur est une unité complète, à double face, du langage de connotation» est une erreur. L'idée hjelmslévienne, pour être correcte, doit être reformulée de la façon suivante: «les codes connotatifs présupposent les codes dénotatifs $\% .{ }^{58}$

Mais la première proposition découle logiquement du modèle hjelmslévien. Parler en terme de codes, c'est se dégager sur le fond du modèle de Hjelmslev, c'est ni plus ni moins que changer de paradigme. Si les codes connotatifs ne font que présupposer les codes dénotatifs, ceux-ci ne sont peut-être alors que le code 
connoté d'un autre plan de dénotation; inversement un code de connotation peut être le dénoté d'un autre; on voit que c'est désormais en termes de systèmes qu'il faut réfléchir, et non plus en termes d'unités bifaces.

Si nous nous tournons enfin spécifiquement vers les catégories de connotations qui seraient de nature idéologique selon KerbratOrrechionni, on trouve la liste suivante : 59

1) les valeurs axiologiques (attitude évaluative de l'énonciateur vs objet dénoté) ;

2) toutes les connotations stylistiques (dans une certaine mesure);

3) les idéologèmes de dénominations particulières ;

4) le « code de référence » : c'est-à-dire les valeurs sémantiques qui renvoient à des systèmes de stéréotypes collectifs ;

5) la plupart des connotations symboliques;

6) certaines métaphores évolutives historiquement.

Selon Kerbrat-Orrechionni, toutes les valeurs qui justifient les évaluations d'un énonciateur renvoient à un système idéologique. De même, tous les styles renvoient à une valorisation d'un certain type de discours. Troisièmement, qualifier les sans-emplois de "demandeurs d'emploi», de "main-d'œuvre inactive», ou de "chômeurs" nous informe sur l'appartenance idéologique du locuteur. Le quatrième point vient de Barthes, il s'agit de tous les "vulgarismes», de toutes les «idées reçues» ${ }^{60}$. Le cinquième point est intéressant puisqu'il s'identifie pratiquement avec les symboles mythiques. Le dernier renvoie aux métaphores dont on peut analyser l'évolution en rapport avec un changement idéologique spécifiable; par exemple «moissonner» qui, dans le passage du monde agraire au monde urbain, ne connote plus l'abondance, mais d'abord le labeur pénible puis ensuite un bucolique retour à la nature.

Tous ces éléments sont pertinents, mais la méthode employée par l'auteur est significative par sa faiblesse. De caractère purement symptômale, cette méthode consiste à collecter, de-ci de-là, tout ce qui, de près ou de loin, semble indiquer la présence du social, du

59. Ibid., pp. 216 à 218.

60. $S / Z$, op. cit., p. 104. 
politique. Mais aucun critère précis d'identification n'est fourni : on se fie au «bon sens», à la compétence idéologique de chacun. Cette défectuosité découle de la position de secondarité de la connotation, de sa nature quasiment anecdotique; une fois exclu du centre, le social revient à la pièce dans ce qu'il a de plus évident. Mais l'aveu suivant de Kerbrat-Orrechionni montre mieux que toute critique la faiblesse congénitale de l'approche:

Les connotations idéologiques constituent un sous-ensemble des faits de connotations. Mais inversement, une idéologie peut fort bien s'exprimer par les moyens du langage dénotatif. ${ }^{61}$

En effet, si l'idéologie peut s'exprimer dans la dénotation même, alors que la distinction de la dénotation et de la connotation a justement pour fonction d'exclure des effets de langage comme ceux produits par l'idéologie, alors la distinction de la dénotation et de la connotation perd sa raison d'être et cesse d'être pertinente. Ou plutôt, là où elle l'est encore, ce n'est plus du langage ou de la langue dont il est question, mais des langages scientifiques les plus formalisés. Bref, admettre que l'idéologie puisse s'introduire dans la dénotation, c'est perdre le dernier avantage que cette distinction conceptuelle comportait ; c'est admettre que le concept de connotation ne peut rendre compte de l'idéologie.

Ce qu'il fallait démontrer! Nous serons donc plus bref en ce qui concerne les approches qui, de près ou de loin se rattachent au saussurisme, dans la mesure où leurs positions sur ces questions s'accordent avec l'essentiel du schéma de secondarité connotative de l'idéologique.

\section{Sémiologie de la communication}

La sémiologie de la communication est fille de la pensée de Saussure. Mais contrairement à la sémiologie barthésienne, elle se préoccupe des codes non linguistiques uniquement en tant que moyens de communication. Quoique Prieto soit plus nuancé ${ }^{62}$, les

61. Ibid., p. 218.

62. Dans «La sémiologie », - in Le Langage, Paris, La Pléïade, 1968, 1521 p., pp. 93-144 -, Prieto accept la possibilité d'une sémiologie de la signification, mais il met la priorité sur la sémiologie de la communication qui serait pour la sémiologie de la signification un meilleur cadre de référence que la linguistique; aussi ne s'occupe-t-il que de celle-ci, puisqu'il la conçoir comme un préalable à la seconde. 
auteurs de cette école conçoivent essentiellement la sémiologie comme la science des codes de communication non linguistiques. Prieto n'affirme-t-il pas que toute sémiologie des fonctions non communicationnelles des codes, « se confondrait, selon nous, avec les sciences de l'homme considérées dans leur ensemble $\gg .{ }^{63} \mathrm{Ce}$ point de vue découle de la conception instrumentaliste du langage de Buyssens, plus précisément, comme ce dernier écrit : «Puisque la fonction primordiale du langage est d'influencer l'entourage, il faut définir le langage comme un moyen d'agir sur l'auditeur, non comme un moyen d'expression. » ${ }^{64}$ Si pour lui la nature du langage est essentiellement sociale, le social n'est conçu que du point de vue de la convention:

Du point de vue social, le langage nous apparaît comme un moyen conventionnel d'agir sur autrui; il est incapable d'exprimer ce qui est concret; si nous pensions dans une langue, nous serions incapables de concevoir le concret. Mais ce caractère abstrait du langage en fait un instrument adéquat pour la science. ${ }^{65}$

Même chose du point de vue du locuteur qui peut ainsi «objectiver ses pensées », et de celui de l'auditeur qui peut se faire ainsi « un portrait psychologique du locuteur ». Si le langage est un instrument de communication, alors la sémiologie sera forcément l'étude des instruments non linguistiques de communication. ${ }^{66}$

Prieto s'engage dans cette voie pour limiter la sémiologie à l'étude des codes de signaux, et pour définir l'acte sémique réussi comme l'usage reconnu d'un signal conventionnel communément partagé par une communauté d'émetteurs-récepteurs. ${ }^{67}$ Le sème (= signe) conserve son caractère bifacial et continue d'obéir au principe de double articulation, bien que diverses combinaisons

63. Dans Pertinence et pratique, Paris, Minuit, 1975, 172 p.; p. 11. Ce point de vue est doublement fautif à notre sens : en effet, il suppose que les sciences humaines se réduisent à l'étude des processus sémiotiques (excluant ainsi toute « physique sociale»), d'autre part il limite indûment le champ propre de la sémiologie à un seul de ses aspects.

64. "Le langage et la logique - Le langage et la pensée ", in Le langage, op. cit., pp. 76-90, p. 77.

65. Ibid., p. 89

66. Ce point de vue, bien qu'intéressant à certains égards, justement en ce qui concerne la communication, nous semble exagérément instrumentaliste; et son point de départ mènera forcément à exclure les formes non communicationnelles de signifiance.

67. Loc. cit. 
articulatoires lui permettent de distinguer divers types de codes sémiologiques: codes de simple articulation de premier ou de second type, codes à double articulation non linguistiques, etc. La différence essentielle entre les codes non linguistiques et le code linguistique repose en ceci que dans les premiers, les signifiés sont radicalement distincts, alors que le second supporte également des cas de regroupements ou d'intersections des signifiés. Ce dernier point accentue encore les limitations que s'impose la sémiologie de la communication. Si Mounin réussit adéquatement à "vérifier si les systèmes de communication non linguistiques ne sont pas tenus pour plus marginaux qu'ils ne le sont réellement " ${ }^{68}$, et à nous convaincre avec force détails de l'importance de l'étude d'objets sémiologiques tels le code de la route, la communication chez les abeilles ou les blasons, il ne parvient pas à justifier plus avant la thèse centrale de cette école : qu'il faille limiter les études sémiologiques à ce type particulier de phénomènes. ${ }^{69}$

On conçoit alors aisément les difficultés que rencontreront ces auteurs pour aborder des problèmes comme celui des systèmes idéologiques. Prieto dans un chapitre de Pertinence et pratique aborde sous le titre de «Pertinence et idéologie » le problème du traitement sémiologique du pouvoir symbolique et de l'idéologie. Son point de départ est essentiellement épistémologique. Considérant l'acquis fondamental de la phonologie praguoise, Prieto affirme que l'identité sous laquelle un sujet connaît une réalité matérielle n'est pas présente dans les objets eux-mêmes, mais résulte d'une pratique d'appropriation, qui établit ce que le sujet considère comme étant les traits pertinents qui permettent de définir les classes d'objets, et donc les concepts sous lesquels ils seront dès lors connus. Ainsi écrit-il :

C'est à son adéquation non pas à l'objet, mais au point de vue duquel on considère celui-ci et d'où dépend sa pertinence, que se mesure la vérité d'un concept ; autrement dit, si un concept peut être considéré comme plus ou moins vrai, c'est dans la mesure où il approche plus ou moins de l'idéal qui consiste à retenir, de l'objet, tout ce qui y est pertinent pour le point de vue sur lequel il se fonde, et cela seulement qui est pertinent pour ce point de vue. ${ }^{70}$

68. Georges Mounin, Introduction à la sémiologie, Paris, Minuit, 1970, 248 p.; p. 18.

69. Chez Peirce notons qu'ils correspondent aux systèmes limités de légisignes indiciels.

70. Op. cit., pp. 143-176; citation p. 146. 
Toute connaissance est une construction significative et toute pratique est une connaissance, donc toute pratique est construction significative. C'est au sens défini plus haut qu'on pourra parler de connaissance objective de la réalité matérielle. Toute connaissance qui concerne les façons dont le sujet connaît les réalités matérielles, c'est-à-dire ses praxis, relève du champ des sciences de l'homme.

Sera nommé idéologie tout discours sur la connaissance de la réalité matérielle qui vise à naturaliser celle-ci, c'est-à-dire à la faire apparaître comme conséquence nécessaire de l'objet luimême, et non de son appropriation historique. De même, sera appelée «connaissance idéologique » de la réalité matérielle toute connaissance naturalisée de cette réalité. Cette naturalisation se reconnaît à son caractère d'évidence. Or, le " pouvoir symbolique » qui régit la vie des groupes sociaux ne confère la légitimité qu'à certains points de vue déterminés, d'où la "pertinence » reconnue est un fait social; cela devient manifeste lorsqu'on étudie les processus de socialisation et les phénomènes de rejet de l'innovation en matière de pertinence. Il ne peut donc pas y avoir de conception de la réalité matérielle ou de conception de la pratique humaine qui soit « neutre ». Inversement le passage de la connaissance idéologique, naturalisée, à la connaissance scientifique, historicisée, de cette connaissance et de son objet, crée une science particulière de l'homme. Les sciences de l'homme, parce qu'elles se trouvent au centre de débats sociaux, ne peuvent échapper aux forces sociales qui ont intérêt à définir la pertinence en un certain sens, comme à naturaliser cette connaissance.

Or la réalité sur laquelle s'exerce la praxis sociale est celle que nous avons désignée comme la réalité historique, et le changement social, qu' elle vise à produire, n'est rien d'autre que la substitution d'une façon de connaître la réalité matérielle à une autre façon de connaître cette réalité. La praxis sociale supposant aussi la connaissance des connaissances de la réalité matérielle, les sciences de l'homme apparaissent comme la condition d'une telle praxis. ${ }^{11}$

$\grave{A}$ vrai dire on sent chez Prieto cette tendance à identifier sémiologie et science de l'homme puisque ce qui le concerne est un mode de connaissance signifiant (et pratique) de l'établissement des critères de pertinence, alors que les sciences «objectives » (en

71. lbid., PP. 164-165. 
son sens précis définit plus haut) s'occupent de la réalité matérielle elle-même. Or, il semble bien que pour lui, ce qui distingue la science en général de l'idéologie est la reconnaissance de l'historicité de la connaissance ; si les classes dominantes résistent à l'établissement de cette historicité, c'est qu'elles ont intérêt à faire apparaître la leur propre comme un fait de nature découlant des réalités matérielles elles-mêmes.

Par cette position peu orthodoxe dans ce contexte, et même étonnante si l'on considère les positions de l'auteur sur la sémiologie, la question de l'idéologie se trouve déplacée et retirée du terrain sémiologique (en son sens) ; elle devient une question épistémologique. Or sur ce terrain, Prieto ne se distingue guère de la tradition qui oppose science à idéologie, sauf sur ce point précis qu'il historicise la connaissance scientifique et fait de l'idéologie une connaissance particulière. En outre, il établit la connaissance comme un enjeu social et politique, mais en cela il ne se distingue pas du marxisme, ce qu'il reconnaît d'ailleurs indirectement en s'appuyant sur la pensée de Marx et la théorie de la praxis. De notre point de vue, il s'agit ni plus ni moins que d'un aveu de l'impossibilité de poser la question de l'idéologie comme une question sémiotique, dans le contexte de la sémiologie de la communication.

Comme le dit Calvet à propos de toute cette école :

L'ennui est que les héritiers de Saussure, conformistes jusqu'au bout, ont tenté de réaliser cette sémiologie de façon aussi réductrice et scientiste qu'ils avaient réalisé leur linguistique : de Buyssens à Priéto ou à Mounin, ce sont les codes qui sont étudiés en priorité, codes confondus, comme je l'ai expliqué, avec la communication, mais surtout codes dénués d'intérêt parce que dénués de toute profondeur sociologique ou psychologique, codes abstraits dont on ne montre jamais comment ils fonctionnent en contexte social. ${ }^{72}$

Les codes dont nous entretiennent les auteurs cités par Calvet, par la méthode trop restrictive qui mène à leur établissement, sont incapables de couvrir adéquatement les objets psychologiques ou sociaux. En outre, la conception de la communication qui en 
découle, réduit tout mode de communication ambigu à une perversion de la référence du signifié. Leur utilité est réelle mais bien délimitée.

Départements de philosophie

$U Q A M / C e ́ g e p d u$ Vieux Montréal 